\title{
OPTIMIZING LOW-COST UAV AERIAL IMAGE MOSAICING FOR CROP GROWTH MONITORING
}

\author{
Pranavan Bupathy ${ }^{1, *}$, Ramesh Sivanpillai ${ }^{2}$, V.V. Sajithvariyar ${ }^{1}$, V. Sowmya ${ }^{1}$ \\ ${ }^{1}$ Center for Computational Engineering and Networking, Amrita School of Engineering, Amrita Vishwa Vidyapeetham, Coimbatore, \\ TN 641112, India (ORCID: 0000-0001-6329-1384) - pranavbupathy1@ gmail.com, (vv_sajithvariyar,v_sowmya)@cb.amrita.edu \\ ${ }^{2}$ Wyoming GIS Center, University of Wyoming, Laramie, WY 82072, USA (ORCID: 0000-0003-3547-9464) - sivan@uwyo.edu
}

KEY WORDS: Precision agriculture, Drones, Aerial images, Image Alignment, Mosaics.

\begin{abstract}
:
High spatial resolution images acquired with drones can provide useful information to farmers for devising suitable management practices and increase crop yield. Data collected as individual frames or images have to be mosaiced using pattern recognition and matching process. Most flight missions collect hundreds of photos with high overlap and side overlap in order to generate mosaic without data gaps or distortion. These frames are aligned using the location information associated with each image. The same features are identified in multiple frames for generating the mosaic. In this process, it is common to use all or most of the images which requires a lot of resources. Uploading and processing hundreds of images could take several hours to days. Many farmers and crop consultants in developing countries may not have the necessary resources to upload hundreds of images. This study assessed the optimal number of images required to generate an image mosaic for a crop field without any data gaps or distortion. Images were collected at two different heights and directions. First, the mosaic was generated using all (100\%) frames followed by subsets containing $90 \%$, through $50 \%$ of images. Results obtained will assist us to plan the settings in future flight missions for acquiring optimal number of images required for generating image mosaic.
\end{abstract}

\section{INTRODUCTION}

Unmanned aerial vehicles (UAVs) or drones are used for a range of applications such as inspection (Chan et.al., 2015), surveying (Meouche et.al., 2016), mapping (Seibert et.al., 2014), crop monitoring and mapping (Raeva et.al., 2019), security (Birk et.al., 2011), identify epiphytes (Anivilla et.al., 2020; Aswin etl.al, 2021), emergency response (Sanjana et.al., 2020). In agriculture drones are used for monitoring crop growth (Tao et.al., 2020), monitoring irrigation issues, pesticide application (Yinka-Banjo and Ajayi, 2020). Drones acquire high spatial resolution images along with other variables and provide rich data that can be used for modelling crop growth (Li et.al., 2016). These data have helped farmers to adapt suitable management to improve crop growth.

Drones that can collect imagery data in visible and infrared regions of the spectrum. Infrared regions are relatively more expensive than those that collect data in the visible spectrum. Similarly, fixed wing drones are relatively more expensive than several quadcopter (four rotor) models. Hexa- and Octa-copter models are required to carry the heavier sensors used for collecting detailed data are expensive. In addition to the platforms and sensors, specialized software is required to process the imagery data collected on the ground. These software are available for both desktop and on online platforms where users have to upload individual images acquired for an area of interest and generate a mosaic, i.e., a single image that will cover the entire study area. These mosaics will show variations in the growth and health in a crop field.
Image registration technique is the basis for combining two or more images (Ait-Aoudia et.al., 2012). These techniques find the distinct key point or feature vector in that image and also find the same feature points in other images to align the photos. When many photos are acquired or at very high spatial resolution, large computing resources are required for creating image mosaic (Carrio et.al., 2017). For very large imagery data volume, cloud-based processing solutions can be rented for remote computation. Based on the computing resources the processing time can take between few hours and days.

Farmers in most developing countries might not have access to recent drone and sensor technologies, and facilities to process aerial images for generating image mosaics of their fields. Recently introduced low-end (lower cost), quad-copter drones, have high quality sensors that are capable of acquiring high spatial resolution images. Sensors on these drones can acquire high quality images and videos. If mosacis of acceptable quality can be generated using these low-cost drones, more farmers can afford to invest in drones.

Seifert et al., (2019) reported that images acquired at low flight altitudes with higher image overlap resulted in most reconstruction details. This study reported the effect of drone height and image overlap while reconstructing forest images.

The primary objective of this study was to assess whether a commonly available low-cost drone can be used for acquiring aerial images and generate good quality mosaic for a crop field. The second objective of this study was to determine the optimal number of images required to generate a mosaic without

\footnotetext{
* Corresponding author
} 
distortion and gaps (potholes). The third objective was to evaluate the effect of drone height during image acquisition on generating the mosaic. If acceptable mosaics can be generated with optimal number of images will reduce the processing time and eliminate the need to invest in high end computing systems.

\section{MATERIALS AND METHODS}

\subsection{Image mosaic}

Panorama is the best example for image mosaicking technique (Szeliski et.al., 1994). Two or more images are taken at slightly different times for the study area are combined to form a single image where the entire target area is visible. The mosaic is constructed by aligning the images in order. Image registration techniques extract feature points available in the images, which are termed as the descriptor vector that describes or locate the local key point in the image. These techniques are used in satellite and UAV acquired image processing (Ait-Aoudia et.al., 2012) biomedical (Shao et.al., 2011), and many other applications.

Algorithms such as scale-invariant feature transform (SIFT) and speed up robust feature (SURF) are used in feature points extraction (Lowe, 1999). Other optimized methods such as BRIEF (Binary Robust independent elementary features) (Calonder et.al., 2011) and ORB (Oriented Fast and Rotated Brief) (Rublee et.al., 2012) are used in other applications. These algorithms find the key point in the image and to compute the descriptor vector that helps to focus more on the extreme feature point. The key point is computed by blurring the image with different level of Gaussian blur and are stacked. The stacked blurred images are subtracted to find the key point in the image. Then the descriptive vector is computed by calculating the local neighbourhood to know the surroundings of the key point. Using the descriptor vector from different images, they are aligned based on transformations such as projective, similarity, affine functions. Finally, the overlapping region of two images are stitched (Szeliski et.al., 2006) based on the pixel intensity value (i.e., Gradient domain) (Levin et.al., 2004). Agisoft ${ }^{\mathrm{TM}}$ uses SIFT to identify the key point (Bert, 2018). For entire study, the recommended default values were used: key point limit $=40,000$ and tie point limit $=4000)$. The key point determines the feature point in 2D image and tie point are used to compute $3 \mathrm{D}$ position of the feature.

\subsection{Data collection}

A DJI Spark ${ }^{\mathrm{TM}}$ drone was used to capture aerial images of Brinjal (Solanum melongena) field located in Vedasandur, Tamil Nadu (India). Aerial images were acquired from two different altitudes: 15 and 13 meters. Flight heights were determined based on safe to fly height in order to avoid obstacles such as trees that were planted along the field boundary, utility poles, and other elevated objects (Sajithvariyar et al., 2019). The study area is in a safe to fly zone.

Hammer $\mathrm{App}^{\mathrm{TM}}$ is an open-source software available for iOS platform, can be used to plan a flight mission over the study area. Hammer $\mathrm{App}^{\mathrm{TM}}$ enables users can input boundary points and other manual waypoints for the field and additional settings like altitude, front and side overlap based on the available flight time. Settings used in this study are summarized in Table 1.

\begin{tabular}{|l|l|l|l|l|l|}
\hline $\begin{array}{l}\text { Heig } \\
\text { ht. } \\
\text { (m) }\end{array}$ & $\begin{array}{l}\text { No. of } \\
\text { images } \\
\left(\begin{array}{l}100 \% \text { of } \\
\text { dataset) }\end{array}\right.\end{array}$ & $\begin{array}{l}\text { Front } \\
\text { overlap } \\
\%\end{array}$ & $\begin{array}{l}\text { Side } \\
\text { Overlap } \\
\%\end{array}$ & $\begin{array}{l}\text { Gimbal } \\
\text { tilt } \\
\text { (deg) }\end{array}$ & $\begin{array}{l}\text { Ground } \\
\text { offset } \\
(\mathrm{m})\end{array}$ \\
\hline $13 \mathrm{~m}$ & 241 & 90 & 52 & -90 & -4.0 \\
\hline $15 \mathrm{~m}$ & 319 & 90 & 52 & -90 & 0 \\
\hline
\end{tabular}

Table 1. Flight settings entered in the Hammer app ( ${ }^{\mathrm{TM}}$ ) for acquiring images for the study area.

The front overlap was set at maximum $(90 \%)$ as it does not affect the flight time. The side overlap settings were adjusted based on the flight time and battery longevity. Flight direction were set at -70 degrees to align the flight path in straight pattern rather than zig-zag pattern as shown in the (Figure 1).

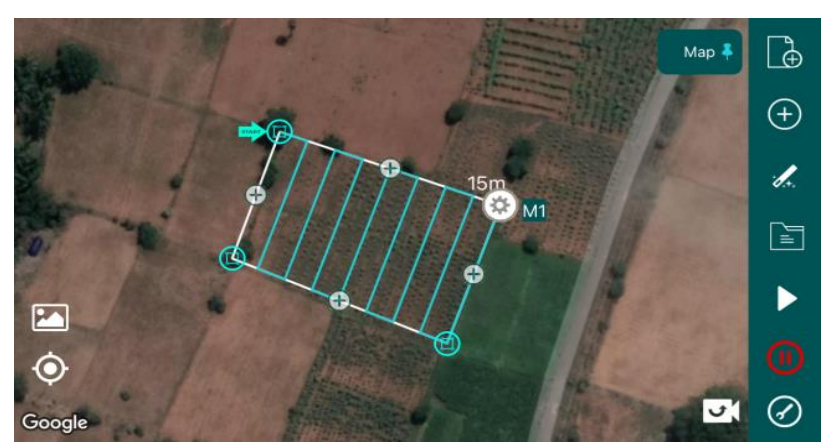

Figure 1. Flight mission plan for the study area set for altitude $15 \mathrm{~m}$ in Hammer app (TM).

Next, the white balance setting in DJI GO4 app was adjusted depending on the flight condition. In this study both missions were flown under sunny conditions (Figure 2).

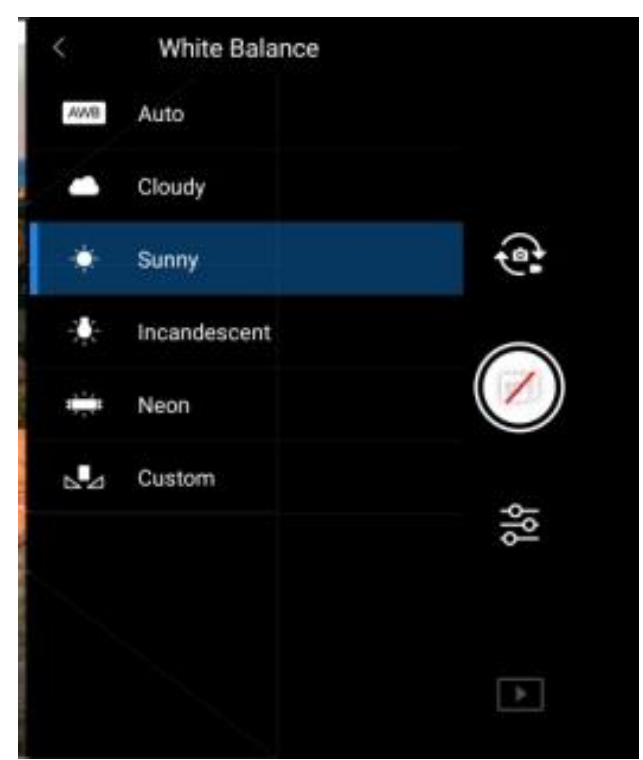

Figure 2. White Balance was set to Sunny in DJI G04 app based on meteorological conditions at the time of the flight.

\subsection{Reference panels}

Black and white reference panels were placed throughout the field (Figure 3 ) in order to determine whether the minimum and 
maximum values change during the mosaic creation process. Methods described by Jeong et al., (2018) was adopted for placing the reference panels. High-end sensors use internal or external methods to calibrate their measurements which are not available for low-end sensors. Minimum (black panel) and maximum (white panel) values after the mosaics generated under different settings will provide insights about the precision of the pixel values in the mosaic.

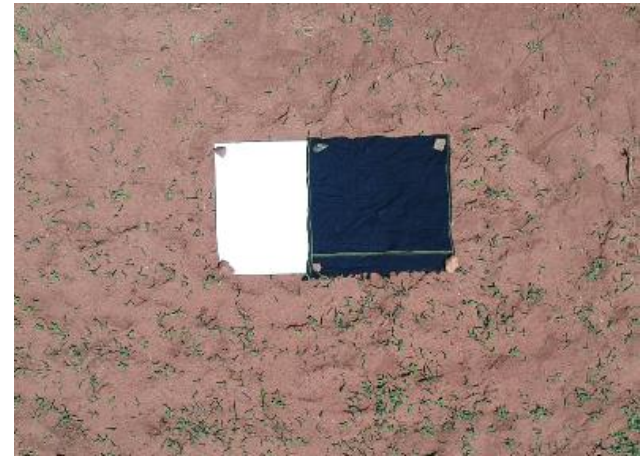

Figure 3. Black and white reference panels placed in the field prior to the flight mission.

\subsection{Mosaic generation}

Leave two blank lines under the key words. Type Aerial images collected from both missions $(15 \mathrm{~m}$ and $13 \mathrm{~m})$ were mosaiced under different settings. The image mosaics were generated with Agisoft software ${ }^{\mathrm{TM}}$ installed in a Windows $10 \mathrm{OS}$, i7 9th generation processor, 8GB RAM and 1660TI graphic card with 6GB VRAM. The process flow for creating mosaic is shown in (Figure 4), The first step was to import all photos that were collected at each flight height.

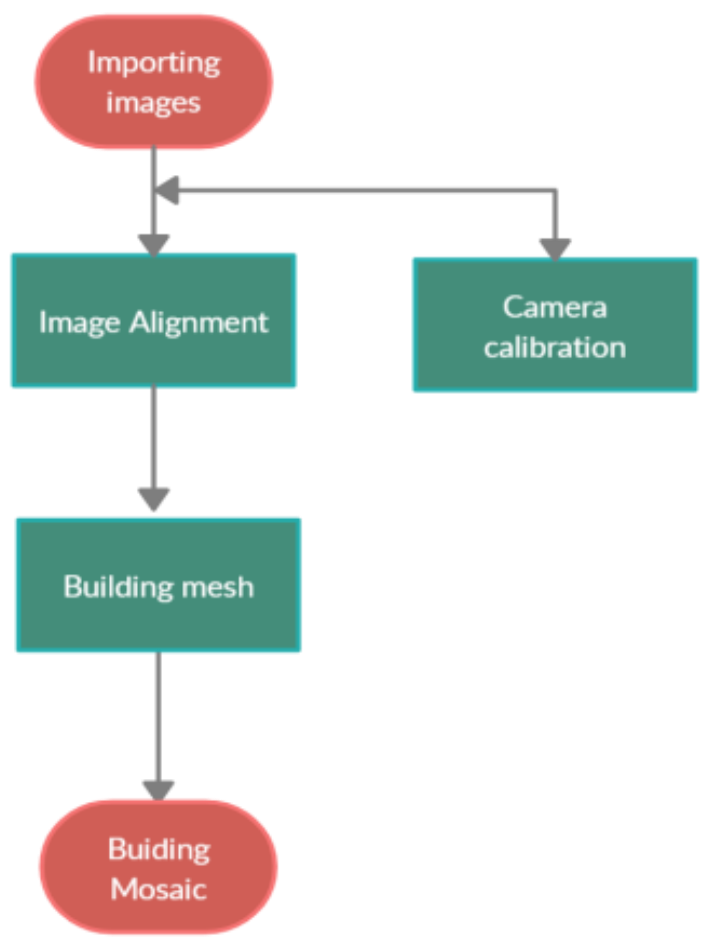

Figure 4. Workflow for building mosaic in Agisoft ${ }^{\mathrm{TM}}$ using aerial images acquired at 13 and $15 \mathrm{~m}$ above the study area.
Camera calibration steps described by (Agisoft, 2011) was applied. Next, the image alignment process and the missing data part were built by meshing process to generate the mosaic. Time taken to generate each mosaic was recorded. The final mosaic products were saved as TIFF files.

The flow diagram shown in (Figure 3) was followed in the present work. The mosaic was generated for each height; each mosaic was created by reducing $10 \%$ of individual images until $40 \%$ or even less until potholes and data loss were noticed. The time taken for alignment of images and mesh building remains the same. This is because the images that were removed to reduce the overlap after the image alignment and mesh were built. So, the change in time will be only based on mosaic building process. Then time needed to generate the outputs (mosaics) at each step was recorded. Following settings were set in Agisoft ${ }^{\mathrm{TM}}$ 's Reduce Overlap tool: capture distance $=40 \mathrm{~m}$; Image overlap $=$ High; Max images $=$ Number of images which is to be removed at each $\%$, for this study the no. of images to be removed is listed in (Table 2).

\begin{tabular}{|c|c|c|c|c|}
\hline $\begin{array}{l}\text { Altitude } \\
(\mathrm{m})\end{array}$ & Dataset $\%$ & $\begin{array}{l}\text { Number of } \\
\text { Available } \\
\text { images }\end{array}$ & $\begin{array}{l}\text { Number of } \\
\text { images to } \\
\text { be } \\
\text { removed }\end{array}$ & $\begin{array}{c}\text { Number of } \\
\text { images } \\
\text { after } \\
\text { removal }\end{array}$ \\
\hline \multirow{7}{*}{13} & 100 & 241 & 0 & 241 \\
\hline & 90 & 241 & 24 & 217 \\
\hline & 80 & 241 & 48 & 193 \\
\hline & 70 & 241 & 72 & 169 \\
\hline & 60 & 241 & 96 & 145 \\
\hline & 50 & 241 & 120 & 121 \\
\hline & 40 & 241 & 145 & 96 \\
\hline \multirow{7}{*}{15} & 100 & 319 & 0 & 319 \\
\hline & 90 & 319 & 32 & 287 \\
\hline & 80 & 319 & 64 & 255 \\
\hline & 70 & 319 & 96 & 223 \\
\hline & 60 & 319 & 128 & 191 \\
\hline & 50 & 319 & 159 & 160 \\
\hline & 40 & 319 & 191 & 128 \\
\hline
\end{tabular}

Table 2. Number of images that were removed at each step.

The quality of mosaic was visually assessed, and the average minimum (black panel) and maximum (white panel) reflectance value was computed for the mosaics generated with different amount $(\%)$ of images acquired for the study area. 


\section{RESULTS}

\subsection{Time required to generate mosaics}

Processing time recorded for each image is a combination of two steps: a) alignment of images and mesh building, and b) mosaic building process. Since the first step was completed with $100 \%$ of images at both elevations (Tables 3 and 4), the processing time for the first step will remain the same for all settings. Differences in time will be based on the mosaic building process step.

\begin{tabular}{|c|c|c|c|}
\hline \multicolumn{2}{|c|}{$\begin{array}{c}\text { Percent \& Number } \\
\text { of } \\
\text { images used }\end{array}$} & Time taken & Tie points \\
\hline 100 & 241 & 57 mins & 0.94 \\
\hline 90 & 217 & 57 mins & 0.94 \\
\hline 80 & 193 & 57 mins & 0.94 \\
\hline 70 & 169 & 56 mins & 0.94 \\
\hline 60 & 145 & 55 mins & 0.94 \\
\hline 50 & 121 & 55 mins & 0.94 \\
\hline 40 & 96 & 54 mins & 0.94 \\
\hline 30 & 72 & 53 mins & 0.94 \\
\hline 20 & 48 & 53 mins & 0.94 \\
\hline
\end{tabular}

Table 3. Time taken to generate mosaics with different percent of aerial images acquired at a flight height of $13 \mathrm{~m}$. All 241 aerial images were used for aligning and mesh building, prior to generating the mosaic.

\begin{tabular}{|c|c|c|c|}
\hline \multicolumn{2}{|c|}{$\begin{array}{c}\text { Percent \& Number of } \\
\text { images used }\end{array}$} & Time taken & Tie points \\
\hline 100 & 319 & 58 mins & 0.68 \\
\hline 90 & 287 & 58 mins & 0.97 \\
\hline 80 & 255 & 55 mins & 0.66 \\
\hline 70 & 223 & 54 mins & 0.67 \\
\hline 60 & 191 & 51 mins & 0.67 \\
\hline 50 & 160 & 50 mins & 0.67 \\
\hline 40 & 128 & 50 mins & 0.67 \\
\hline
\end{tabular}

Table 4. Time taken to generate mosaics with different percent of aerial images acquired at a flight height of $15 \mathrm{~m}$. All 319 aerial images were used for aligning and mesh building, prior to generating the mosaic.

Removal of photos reduced the processing time, but it was not linear and in few instances the time taken remained the same (Tables 3 and 4). Images from both datasets acquired at 13 and 15 meters aligned without any noticeable problems. For aerial images acquired at $13 \mathrm{~m}$, the tie point identification was $94 \%$ at each removal. However, for $15 \mathrm{~m}$ the tie point varied from $66 \%$ to $97 \%$.
Based on visual inspection, mosaic generated with $100 \%$ of images $(n=241)$ acquired at 13 meters appeared to be of high quality (Figure 5). No noticeable distortion, loss or potholes were noticed until $60 \%$ of the images $(n=145)$ were used for generating the mosaic.

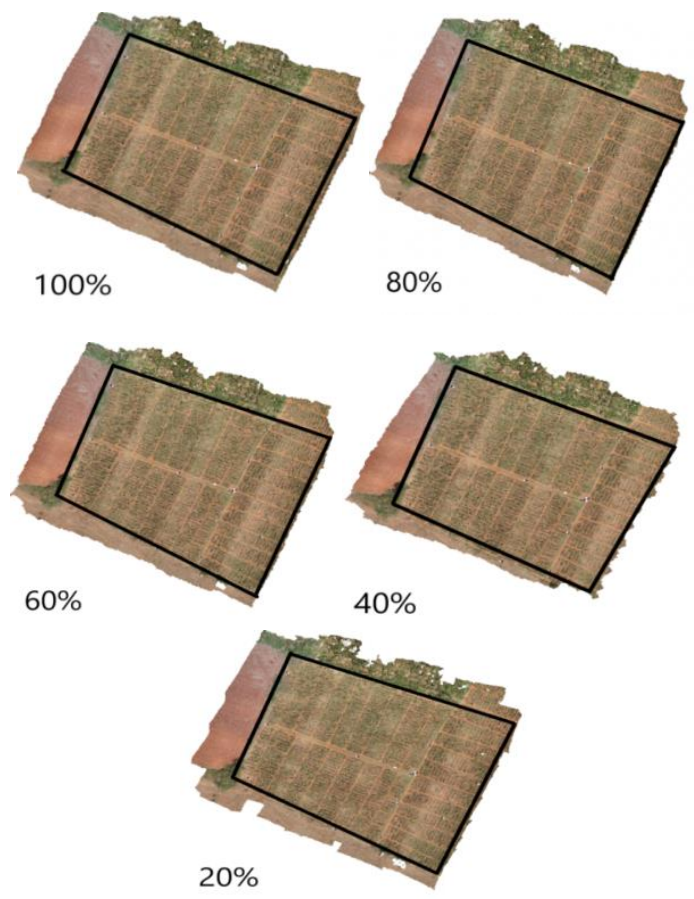

Figure 5. Mosaic generated with 100\%, 80\%, 60\%, 40\% and $20 \%$ of aerial images acquired at a flight height of $13 \mathrm{~m}$.

Loss of information was noticed when $40 \%$ of the images $(n=$ 96) and there were more gaps in the mosaic generated with $20 \%$ of the images.
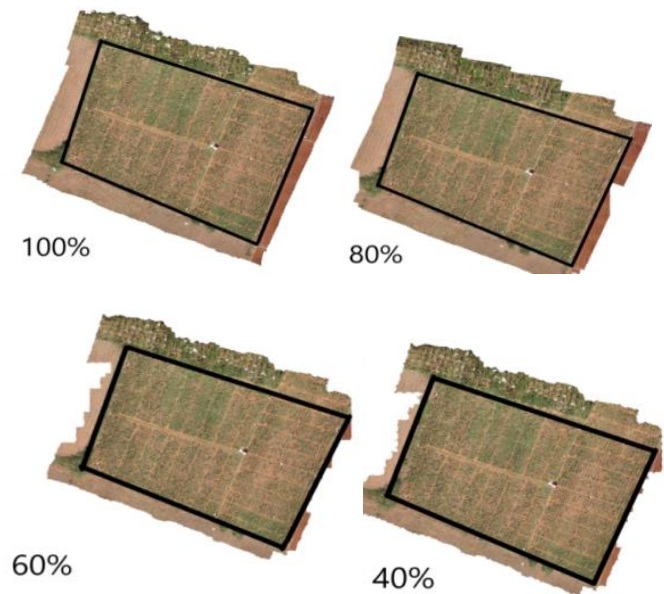

Figure 6. Mosaic generated with $100 \%, 80 \%, 60 \%$, $40 \%$ of aerial images acquired at a flight height of $15 \mathrm{~m}$.

Mosaic generated with $100 \%$ of images $(n=319)$ acquired at 15 $\mathrm{m}$ appeared to be of high quality (Figure 6). When $20 \%$ of the images were eliminated, minor loss was noticed in the south eastern corner of the field. Removing additional $20 \%$ of images in each step resulted in increasing loss in information. When 
only $40 \%$ of the images were used, several gaps along the field boundary.

\subsection{Changes in the reference panel values}

The minimum (black panel) and maximum (white board) values were obtained for all reference panels in each mosaic generated with $100 \%, 80 \%, 60 \%, 40 \%$ and $20 \%$ of aerial images acquired at $13 \mathrm{~m}$ and $15 \mathrm{~m}$ are presented in Figures 7 and 8 respectively.
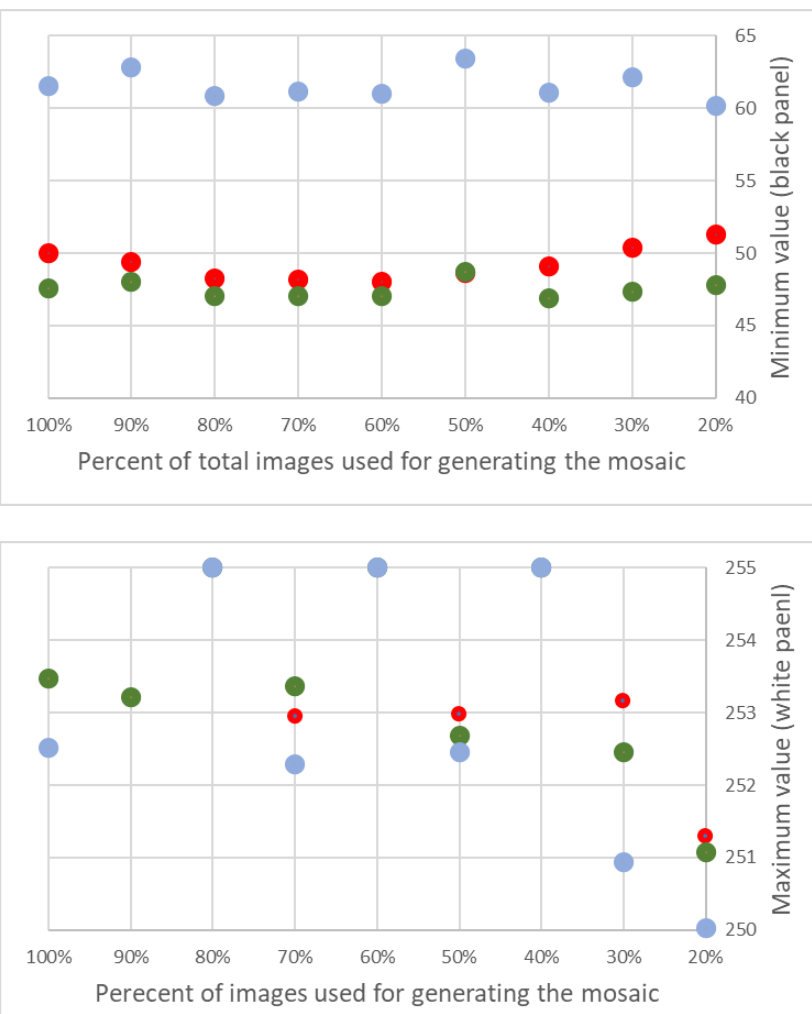

Figure 7. Minimum, black panel (above) and maximum, white panel (below) values measured in the blue, green, and red bands

(as colored dots) from the mosaics generated with different percent of aerial images acquired at 13 meters.

The minimum (black panel) values recorded from the mosaics generated with aerial photos acquired at 13 meters, revealed that they were above zero in all three (RGB) bands (Figure 7 above). Minimum values in the blue band (blue dots) were higher in comparison to the red (red dots) and green (green dots) bands. The maximum (white panel) values varied between 250 and 255 for all the RGB bands (Figure 7 below). Unlike the minimum values, the maximum values did not show any distinct pattern across the three (RGB) bands.

The minimum (black panel) values recorded from the mosaics generated with aerial photos acquired at 15 meters, revealed that they were also above zero in all three (RGB) bands (Figure 8 above). Minimum values in the blue band (blue dots) were higher in comparison to the red (red dots) and green (green dots) bands. The maximum (white panel) values varied between 251 and 255 for all the RGB bands (Figure 8 below). Unlike the minimum values, the maximum values did not show any distinct pattern across the three (RGB) bands.
Minimum and maximum values in the mosaics generated with different number of aerial images acquired at 13 and 15 meters showed random variations, instead of increasing or decreasing patterns. In other words, the number of photos used for generating the mosaic will not affect the minimum or maximum values. It is also evident that the minimum values were well above zero.
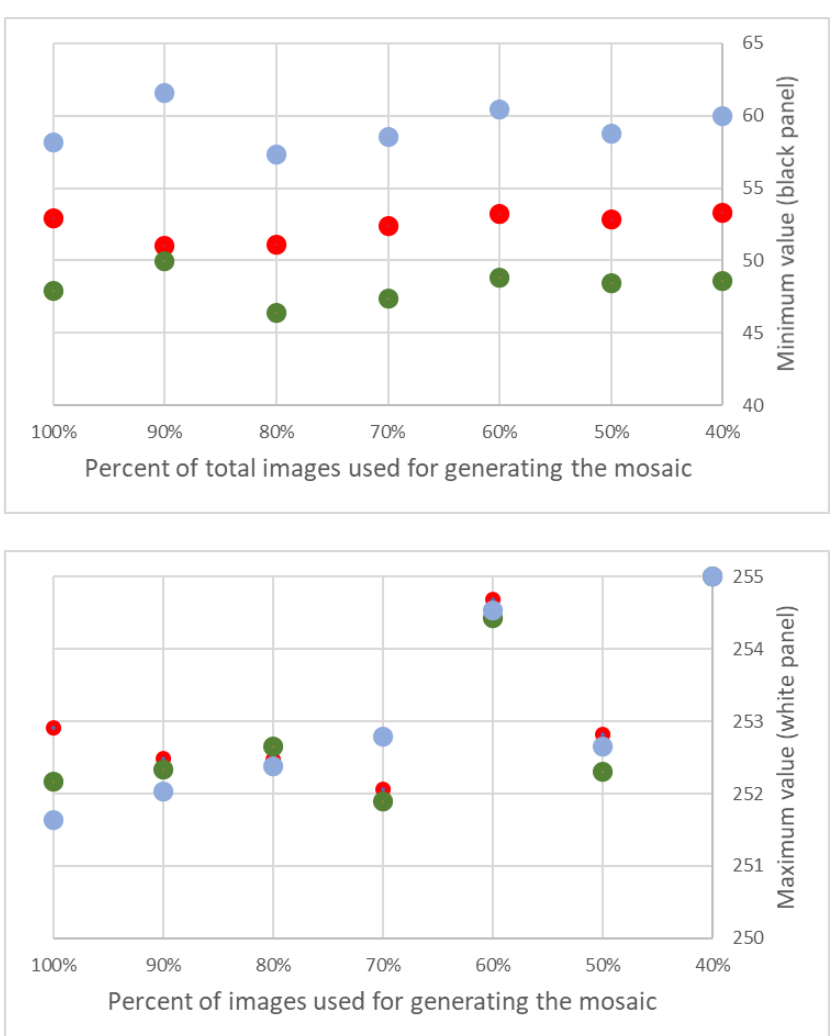

Figure 8. Minimum, black panel (above) and maximum, white panel (below) values measured in the blue, green, and red bands (as colored dots) from the mosaics generated with different percent of aerial images acquired at 15 meters.

Based on these results, we can conclude that elevation at which the images were flown will influence the quality of the mosaic. Mosaics could be generated with relatively fewer number of images (20\%) acquired at 13 meters indicating that the flight height plays an important role. These findings concur with those reported by Seifert et al., (2019).

\section{CONCLUSIONS}

Low-cost drones can be used to generate image mosaics for monitoring crop growth. In this study, a widely available drone was used, but we hypothesize that similar low-cost drones can achieve similar results.

As the number of photos used for generating the mosaic decreased, the time required to generate the mosaic slightly reduced. However, when working with larger number of images considerable time can be saved. However, we recommend users collect the maximum number of images for their study area depending on the drone's flight time. This will ensure that there will be no information loss while generating mosaics with fewer number of images aimed at reducing processing time and computing resources. We recommend that the front overlap can 
be set as high as $90 \%$ and it will not change the flight time. Also, we recommend that side overlap can be set at least $50 \%$. Results obtained in this study indicate that it is possible to generate mosaics without gaps and distortions using $80 \%$ of the images.

Relatively fewer number of images acquired from 13 meters can be used to mosaics without gaps and distortions. In contrast, more images acquired from 15 meters were required to acceptable mosaics.

\section{ACKNOWLEDGEMENTS}

Authors thank Prof. K. P. Soman, Head, Center for Computational Engineering and Networking (CEN) at Amrita Vishwa Vidyapeetham, Coimbatore, Tamil Nadu for his valuable support.

\section{REFERENCES}

Ait-Aoudia, S., Mahiou, R., Djebli, H., Guerrout., E., 2012: Satellite and Aerial Image Mosaicing - A Comparative Insight, 652-657. doi.org/10.1109/iv.2012.113.

Agisoft, 2011: Agisoft Lens User's Manual (Version 0.4.0). downloads.agisoft.ru/lens/doc/en/lens.pdf (11 April 2021).

Anivilla, S., Sajithvariyar, V. V., Sowmya, V., Soman, K. P., Sivanpillai, R., and Brown, G. K 2020: Identifying epiphytes in drone photos with a conditional generative adversarial network (C-GAN), Int. Arch. Photogramm. Remote Sens. Spatial Inf. Sci., XLIV-M-2-2020, 99-104, doi.org/10.5194/isprs-archivesXLIV-M-2-2020-99-2020.

Aswin, S., Sajithvariyar, V.V., Sivanpillai, R., Sowmya, V., Brown, G.K., 2021: Effect of Annotation and Loss Function on Epiphyte Identification using Conditional Generative Adversarial Network. 2021 International Conference on Advances in Electrical, Computing, Communication and Sustainable Technologies (ICAECT) 1-6. doi.org/ 10.1109/ICAECT49130.2021.9392478.

Birk, A., Wiggerich, B., Bülow, H., Pfingsthorn, M., Schwertfeger, S., 2011: Safety, Security, and Rescue Missions with an Unmanned Aerial Vehicle (UAV). Journal of Intelligent \& Robotic Systems, 64(1), 57-76. doi.org/10.1007/s10846-0119546-8.

Calonder, M., Lepetit, V., Ozuysal, M., Trzcinski, T., Strecha, C., Fua, P., 2012: BRIEF: Computing a Local Binary Descriptor Very Fast. IEEE Transactions on Pattern Analysis and Machine Intelligence, 34(7), 1281-1298. doi.org/10.1109/tpami.2011.222.

Carrio, A., Sampedro, C., Rodriguez-Ramos, A., Campoy, P., 2017: A Review of Deep Learning Methods and Applications for Unmanned Aerial Vehicles. Journal of Sensors, 2017: 3296874. doi.org/10.1155/2017/3296874.

Chan, B., Guan, H., Jun, J., Blumenstein, M., 2015: Towards UAV-based bridge inspection systems: a review and an application perspective. Structural Monitoring \& Maintenance, 2(3), 283-300. doi.org/10.12989/SMM.2015.2.3.283.

Jeong, Y., Yu, J., Wang, L., Haein, S., Koh, S., Park, G., 2018: Cost-effective reflectance calibration method for small UAV images. International Journal of Remote Sensing, 39(21), 7225-

\section{0. doi.org/10.1080/01431161.2018.1516307.}

Levin, A., Zomet, A., Peleg, S., Weiss, Y. 2004: Seamless Image Stitching in the Gradient Domain. Computer Vision. In: Pajdla T., Matas J. (eds) Computer Vision - ECCV 2004. ECCV 2004. Lecture Notes in Computer Science, vol 3024. Springer, Berlin. 377-389. doi.org/10.1007/978-3-540-24673-2_31.

Li, Z., Isler, V., 2016: Large Scale Image Mosaic Construction for Agricultural Applications. IEEE Robotics and Automation Letters, 1(1), 295-302. doi.org/10.1109/LRA.2016.2519946.

Lowe, D.G., 1999: Object recognition from local scale-invariant features. Proceedings of the Seventh IEEE International Conference on Computer Vision, 1150-1157. doi.org/10.1109/ICCV.1999.790410.

Raeva, P.L., Šedina, J., Dlesk, A., 2019: Monitoring of crop fields using multispectral and thermal imagery from UAV. European Journal of Remote Sensing,52(sup1), 192201, doi.org/10.1080/22797254.2018.1527661.

Rublee, E., Rabaud, V., Konolige, K., Bradski, G., 2011: An efficient alternative to SIFT or SURF. 2011 International Conference on Computer Vision, 2564-2571. doi.org/10.1109/iccv.2011.6126544.

Sanjana, P., Prathilothamai, M., 2020: Drone Design for First Aid Kit Delivery in Emergency Situation. Sixth International Conference on Advanced Computing \& Communication Systems. doi.org/10.1109/ICACCS48705.2020.9074487.

Sajithvariyar, V.V., Sowmya, V., Gopalakrishnan, E.A., Soman, K.P., Bupathy, P., Sivanpillai, R., Brown, G.K., 2019: Opportunities and challenges of launching UAVs within wooded areas. Proceedings of the 2019 ASPRS Annual Conference. Denver, CO. USA.

Seifert, E., Seifert, S., Vogt, H., Drew, D., van Aardt., J., Kunneke, A., Seifert, T., 2019: Influence of Drone Altitude, Image Overlap, and Optical Sensor Resolution on Multi-View Reconstruction of Forest Images. Remote Sensing, 11(10), 1252-. doi.org/10.3390/rs11101252.

Shao, H.., Hwang, W., Chen, Y., 2011: Biomedical image mosaicing: An optimized multiscale approach. IEEE International Symposium on Biomedical Imaging: From Nano to Macro. 1374-1378. doi.org/10.1109/isbi.2011.5872656.

Szeliski, R., 1994: Image mosaicing for tele-reality applications. Proceedings of 1994 IEEE Workshop on Applications of Computer Vision. 44-53. doi.org/10.1109/acv.1994.341287

Szeliski, R., 2006: Image Alignment and Stitching: A Tutorial. Foundations and Trends in Computer Graphics and Vision, 2(1), 1-104. doi.org/10.1561/0600000009.

Tao, H., Feng, H., Xu, L., Miao, M., Long, H., Yue, J., Li, Z., Yang, G., Yang, X., Fan, L., 2020: Estimation of Crop Growth Parameters Using UAV-Based Hyperspectral Remote Sensing Data. Sensors, 20(5), 1296. doi.org/10.3390/s20051296.

Yinka-Banjo, C., Ajayi, O., 2020: Sky-Farmers: Applications of Unmanned Aerial Vehicles (UAV) in Agriculture In Autonomous Vehicles, Dekoulis, G. (Ed.). IntechOpen, London, UK. doi.org/10.5772/intechopen.89488. 